\title{
CRONOLOGÍA DEL PERIODO MEDIO EN EL VALLE DE AZAPA, NORTE DE CHILE: ESTILOS, FECHADOS Y CONTEXTOS CULTURALES DEL POBLAMIENTO HUMANO
}

\author{
CHRONOLOGY OF THE MIDDLE PERIOD IN THE AZAPA VALLEY, NORTH \\ OF CHILE: STYLES, DATES AND CULTURAL CONTEXTS OF THE HUMAN \\ SETTLEMENT
}

Iván Muñoz Ovalle

\begin{abstract}
Se presentan los resultados del análisis de 116 fechados radiométricos obtenidos por medio del método de Carbono 14 (C14) y Termoluminiscencia (TL). Los fechados fueron tomados de nueve estudios publicados, desarrollados en el Valle de Azapa. Las muestras datadas fueron obtenidas de entierros (cuerpos) y ofrendas (cerámicas) de cementerios vinculados al periodo Medio. Cronológicamente este periodo ha sido asociado a la presencia del Horizonte Tiwanaku en los Valles Occidentales, 500 al 1000 DC, cuyo rasgo más representativo es la presencia de cerámica decorada con formas de keros, tazones, pucos y jarras. Sin embargo, los fechados obtenidos vinculados con estos estilos cerámicos corresponden mayormente a dataciones tardías, en un rango desde los 900 al 1100 DC cal. Por otra parte, la información reciente obtenida del cementerio Az-115 sobre nuevas dataciones, muestran que en los primeros ocho siglos del primer milenio de la Era Cristiana en el poblamiento azapeño no se observa una presencia Tiwanaku, sino más bien una continuidad aldeana vinculada a una historia local con raíces en el periodo Formativo.

El presente estudio, apunta a: (a) caracterizar los inicios y el desarrollo del periodo Medio en el Valle de Azapa, tomando como indicadores diagnósticos los registros de los cementerios Az-115 y Az-75, los que presentan evidencias de un proceso agrícola aldeano de carácter local, incluso con evidencias relacionadas al periodo Formativo, abarcando el periodo Medio hasta el siglo VIII, y (b) definir una segunda etapa o fase II para periodo Medio en el Valle de Azapa, caracterizada por la consolidación de un poblamiento local aldeano. En esta segunda fase, los estilos corresponden a Cabuza, Maitas y Chiribaya, que caracterizarían los estilos cerámicos locales y habrían coexistido con estilos vinculados con Tiwanaku. Al parecer es en esta etapa en donde las poblaciones locales comienzan a definir una identidad regional en nuestros valles cuya expresión final la observamos en lo que se conoce como Cultura Arica, alfarería que caracteriza el periodo Intermedio Tardío (PIT) en los valles y costa de Arica.
\end{abstract}

Palabras claves: cronología, fechas radiométricas, poblaciones prehispánicas periodo Medio, estilos cerámicos.

The results are presented of the analysis of a set of 116 radiometric dates obtained through the method of Carbon 14 (C14) and thermoluminescence (TL) dating. These datings were taken from nine published studies carried out in the Azapa Valley. Regarding dated samples, these were mostly taken from burials (bodies) and offerings (ceramic) from cemeteries in the Azapa Valley linked to the Middle period. Chronologically this period has been associated with the presence of the Tiwanaku Horizon in the western valleys, 500 to $1000 \mathrm{AD}$, and its most representative feature is the presence of painted pottery with forms of keros, bowls, pucos and jars. However, the datings obtained for these ceramics mostly correspond to late datings within a range from Cal 900 to 1100 AD. On the other hand, recent information obtained from cemetery Az-115 on new dates indicates that in the first eight centuries of the first millennium of the Christian era there is no Tiwanaku presence in the Azapeño settlement, but rather a type of village continuity linked to a local history with roots in the Formative period.

The present study aims: (a) to characterize the beginnings and development of the Middle period in the Azapa Valley, taking as diagnostic elements the site records of cemeteries $A z-115$ and Az-75, which present evidence of a local village agricultural process, including evidence related to the Formative period, spanning the Middle period until the eighth century AD; and (b) to define a second stage or phase II of the Middle period in the Azapa Valley, characterized by the consolidation of a local village settlement. In this second phase, the Cabuza, Maitas and Chiribaya styles would characterize the local ceramic styles and would also coexist mainly with styles related to Tiwanaku. Apparently, this is the stage in which local people began to define their own regional identity, whose final expression can be observed in our valleys in what is known as Arica Culture, pottery that characterizes the late Intermediate period (LIP) in the valleys and coast of Arica.

Key words: Chronology, radiometric dates, pre-Hispanic populations, Middle period, ceramic styles.

\footnotetext{
${ }^{1}$ Departamento de Antropología, Universidad de Tarapacá, Arica, Chile. imunoz@uta.cl
}

Recibido: abril 2018. Aceptado: enero 2019.

http://dx.doi.org/10.4067/S0717-73562019005001801. Publicado en línea: 19-agosto-2019. 
Un análisis sobre la reconstrucción inicial del poblamiento humano del periodo Medio en el Valle de Azapa, señala que este se estructuró sobre la base de formas y estilos de cerámica pintadas, halladas como ofrendas en los entierros de cementerios de dicho valle. Un primerestudio sobre la secuencia alfarera en el Valle de Azapa, fue publicado por Dauelsberg (1961, 1972/1973) (Figuras 1 y 2). Para desarrollar esta periodificación, el autor tomó como antecedente el modelo cronológico planteado por Rowe (1962), sobre secuencias culturales en el área andina, señalando por ejemplo que el Horizonte Medio se encontraría representado por Tiwanaku Clásico en su fase IV; junto a los estilos locales Loreto Viejo, Sobraya y Cabuza; Posteriormente Dauelsberg (1985) plantea que el periodo Medio estaría constituido por el complejo/fase Cabuza, cuyas fechas oscilarían alrededor del 380 DC (Figuras 3 y 4) y Las Maitas que alcanzaría los 700 DC (Figuras 5, 6 y 7). Añade que el estilo Loreto Viejo (Figura 8) correspondería a ejemplares vinculados a la influencia de Tiwanaku, que irían desde la fase clásica hasta la fase expansiva. Desde el punto de vista del poblamiento humano, señala que los grupos que manufacturaron esta cerámica serían colonos altiplánicos que se habrían asentado en el Valle de Azapa con la idea de explotarlo agrícolamente. Respecto a Cabuza, se trataría de una población de origen altiplánica que introdujo una agricultura intensiva en el valle. Plantea finalmente que con las poblaciones Cabuza alrededor del 400 DC se habría iniciado el Desarrollo Regional en los valles de Arica.

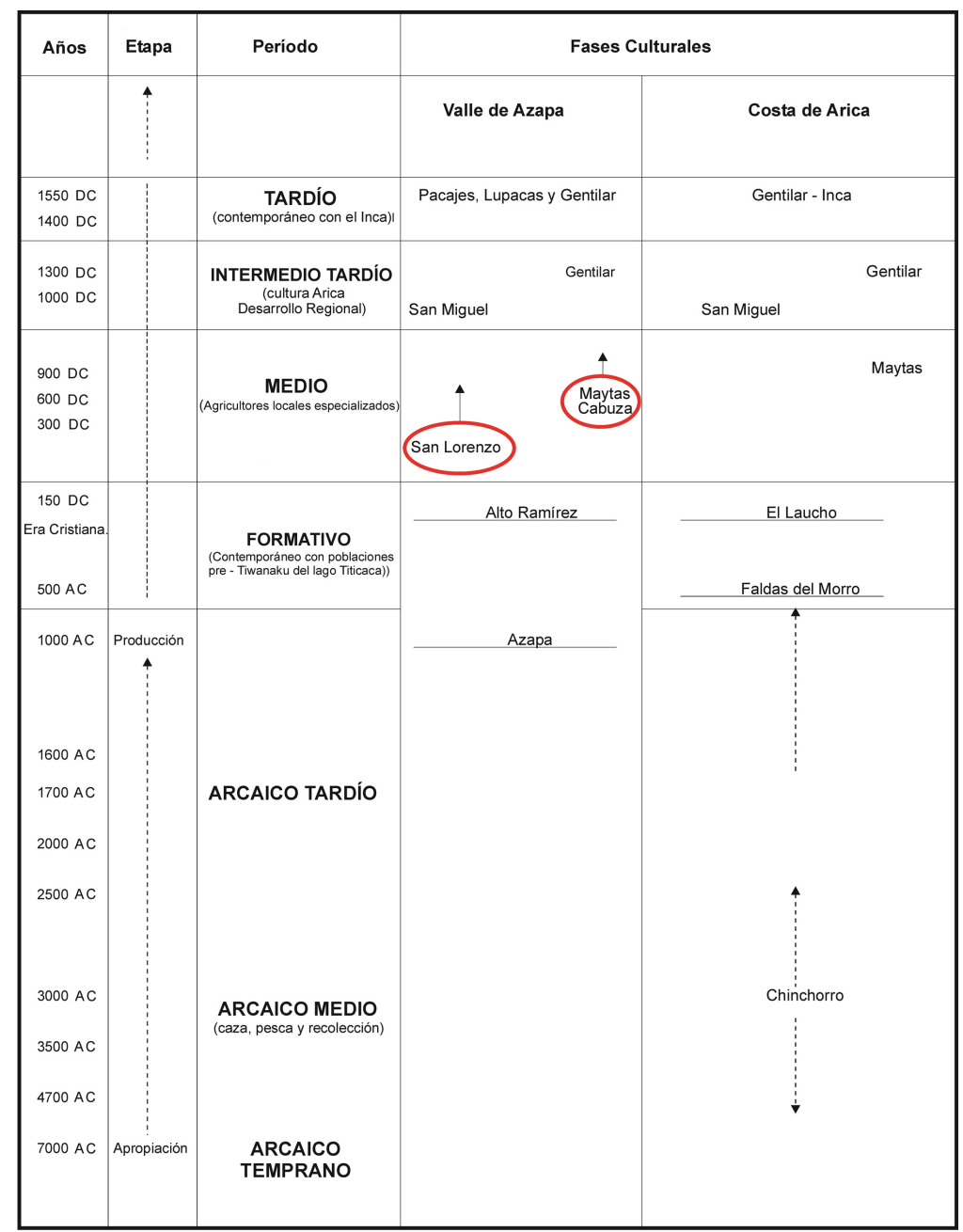

Figura 1. Secuencia cultural prehispánica del Valle de Azapa y borde costero de Arica.

Pre-Hispanic cultural sequence of the Azapa Valley and the coast of Arica. 


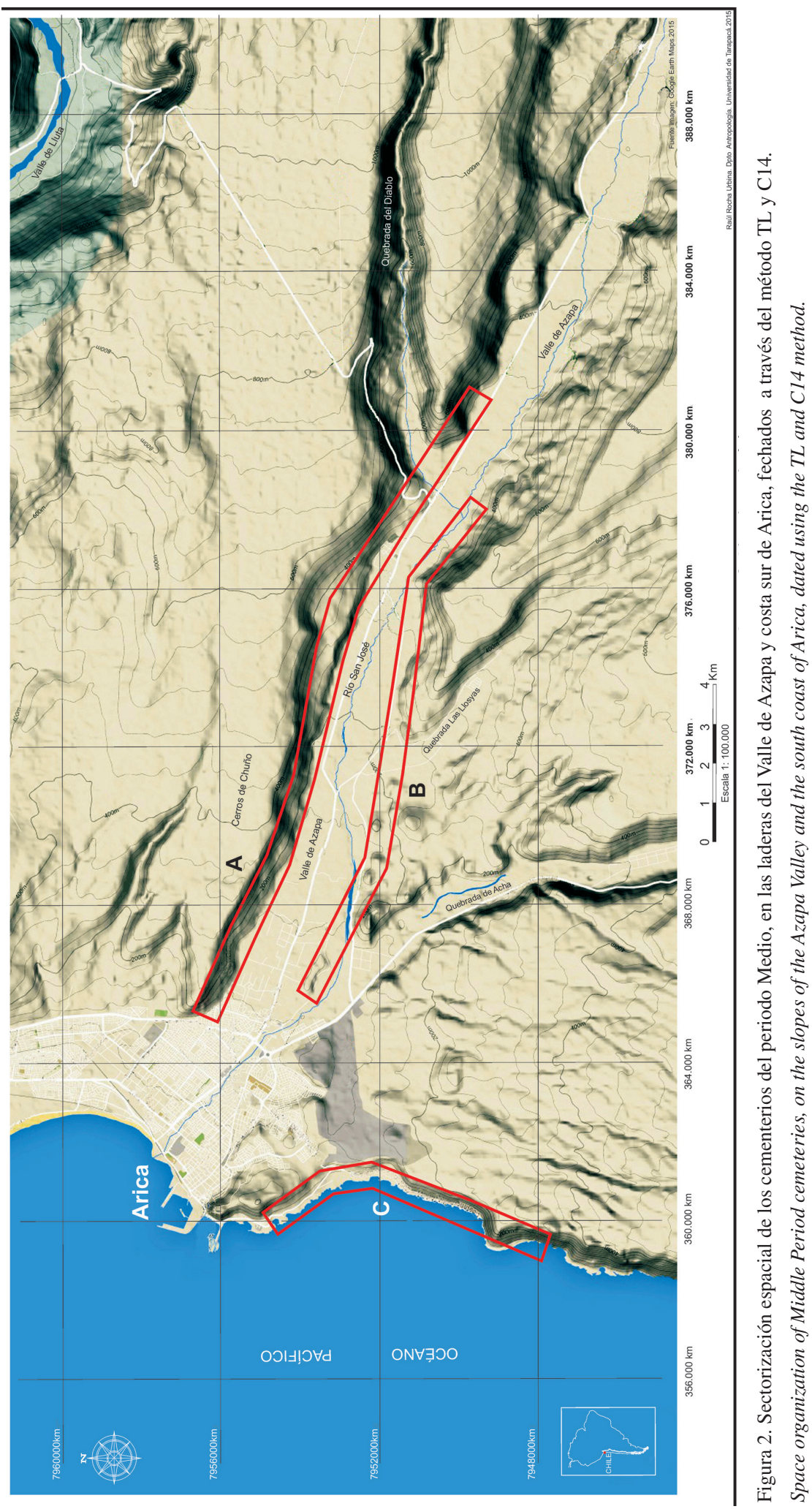




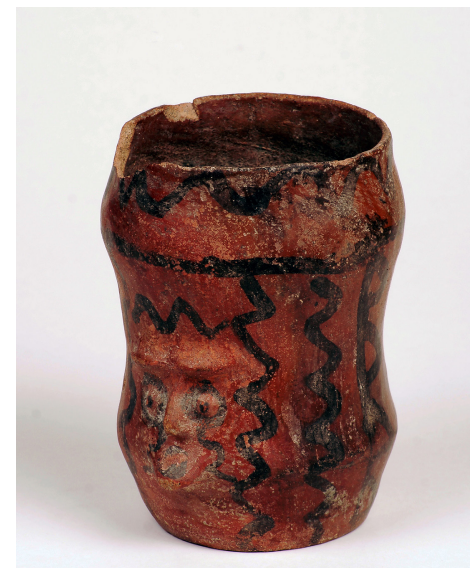

Figura 3. Estilo Cabuza. Vaso decorado con elementos geométricos, lineales serpenteados color negro, sobre engobe rojo. Modelado sobre-relieve representando un rostro humano. Sitio Az-71, tumba $\mathrm{N}^{\circ} 59$.

Cabuza style. Vase decorated with geometric elements, twisted linear in black over red clay. Modeled embossing representing a human face. Az-71 site, tomb $N^{\circ} 59$.

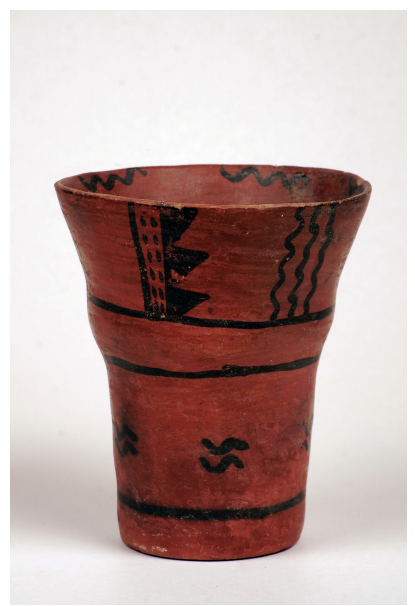

Figura 4. Estilo Cabuza. Vaso kero decorado con elementos geométricos, lineales-triangulares color negro, sobre engobe rojo anaranjado. Sitio Az-1, tumba $\mathrm{N}^{\circ} 1$.

Cabuza style. Kero vase decorated with geometric, linear-triangular elements, black over red-orange clay. Az-1 site, tomb $N^{\circ} 1$.

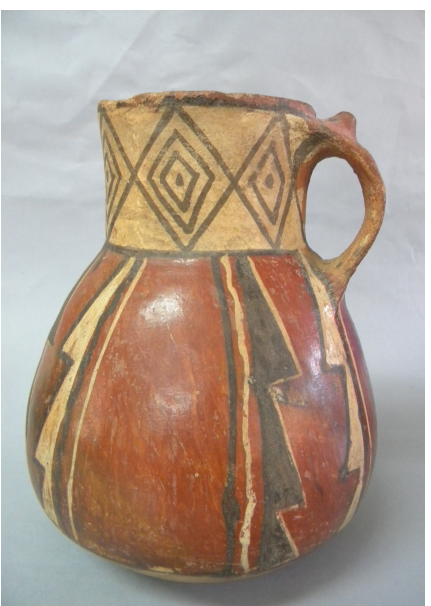

Figura 5. Jarra estilo Las Maitas, Azapa $4 / 175 / 59$.

Jar style, Las Maitas, Azapa 4/175/59.

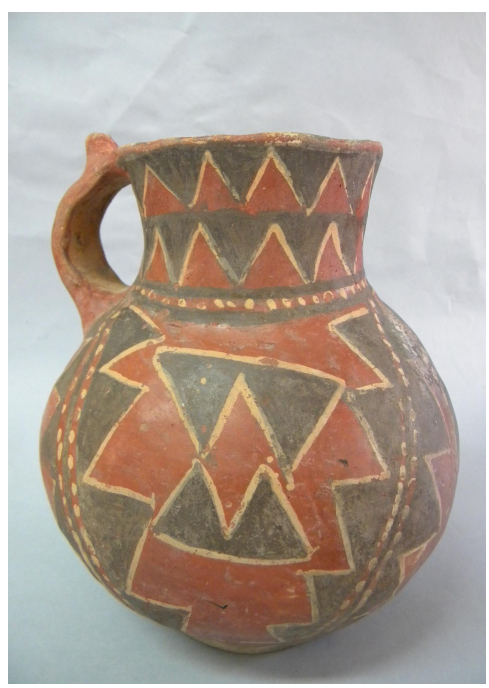

Figura 6. Jarra estilo Las Maitas, Azapa 4/176/59.

Jar style, Las Maitas, Azapa 4/176/59.

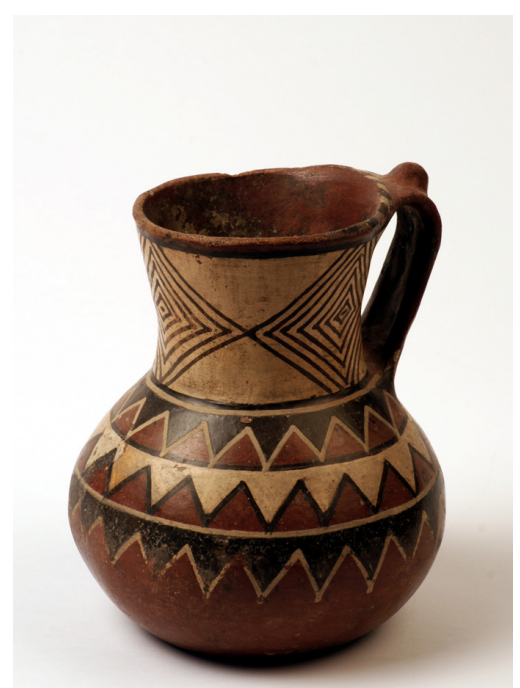

Figura 7. Jarra estilo Maitas, Sitio Az-6. Maitas style pottery, site Az-6.

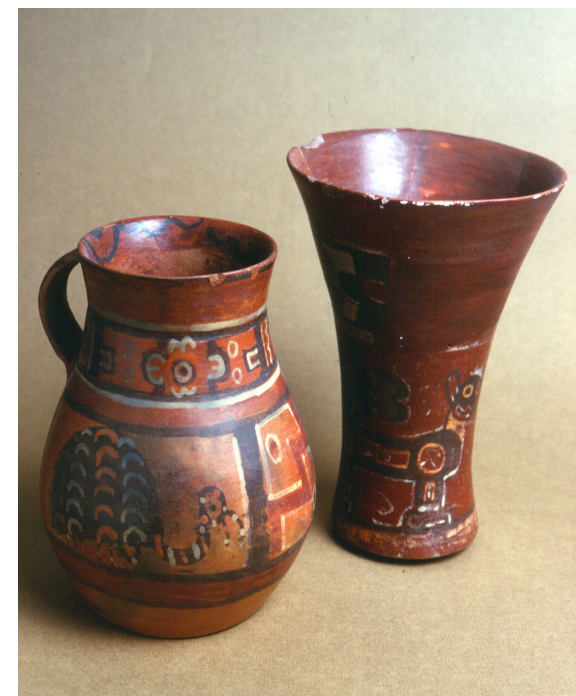

Figura 8. Estilo Loreto Viejo, . Jarra y vaso kero. Sitio Az-6.

Loreto Viejo style pottery, site Az-6. 
Hoy se reconoce que durante el periodo Medio, coexistieron en el espacio y en el tiempo dos tradiciones culturales, las que han sido definidas principalmente a través de la cerámica y tejidos hallados en contextos funerarios: una que se vincularía a la tradición altiplánica y se caracterizaría por los estilos cerámicos Cabuza, Azapa/Charcollo y Tiwanaku, y otra de tradición de Valles Occidentales caracterizada por cerámica Maitas-Chiribaya (Uribe 1999).

Desde el punto de vista de su manufactura, el tipo Cabuza (ca. 500-1200 DC) es una cerámica de pastas compactas principalmente arenosas, de cocción oxidante, con superficies alisadas y revestidas con un grueso engobe rojo y decoración pintada fundamentalmente bicolor (negro y/o blanco sobre revestimiento rojo); este tipo según Uribe (1999) reproduce formas (vasos, jarros, tazones, escudillas y pequeños sahumadores), decoración y patrones tecnológicos altiplánicos.

La cerámica Azapa/Charcollo (600-800 DC) (Figura 9), definida originalmente por Dauelsberg y reformulada por Espoueys et al. (1995), caracteriza una alfarería de aspecto burdo con manchas de pintura roja que, durante el periodo Medio, aparece asociada tecnológica y contextualmente a la cerámica de la tradición altiplánica Tiwanaku y aparentemente es distinta de Charcollo presente durante el Intermedio Tardío en, valles altos y sierra de Arica así como en Tarapacá (Dauelsberg 1972/1973; Espoueys et al. 1995; Muñoz y Chacama 2006; Santoro et al. 2004; Uribe 1999).

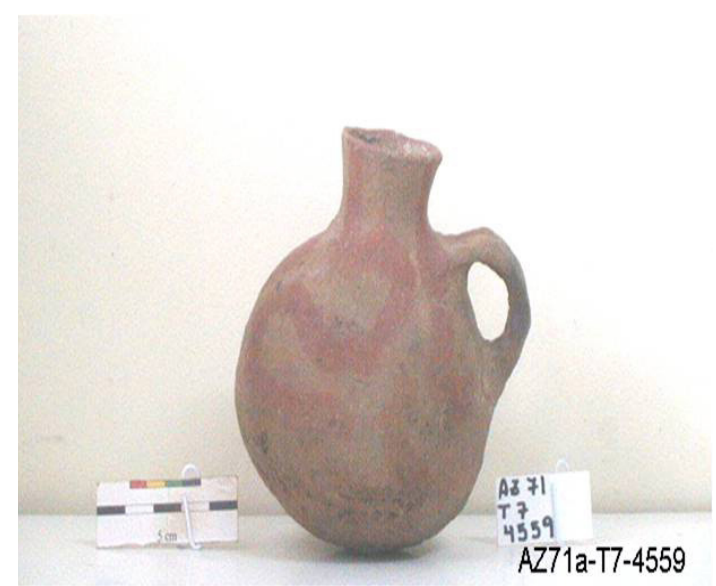

Figura 9. Alfarería estilo Charcollo, sitio Az-71a.T-7, Azapa. Charcollo style pottery, Az-71a.T-7 site, Azapa.
La cerámica Tiwanaku (650-1270 DC) se caracteriza por su policromía (negro, blanco, rojo, violáceo, naranja, sobre revestimiento rojo), manufacturada con pastas muy compactas, arenosas pero homogéneas a coladas, posiblemente locales, cocidas en ambiente oxidante bastante regular, superficies alisadas y revestidas con una delgada capa roja. Muestra una prolija decoración cuya iconografía se basa en Tiwanaku V Expansivo del Lago Titicaca, principalmente en keros, tazones y jarros. De acuerdo a los estudios cerámicos llevados a cabo en las últimas décadas, Cabuza es interpretada como una expresión local influenciada por Tiwanaku en Arica, puesto que copia sus formas y decoración y comparte ciertos atributos con otras cerámicas de los Valles Occidentales del sur peruano. La cerámica de estilo Tiwanaku habría llegado a Arica desde el núcleo de Moquegua, sur peruano (Espoueys et al. 1995; Uribe 1999; Uribe y Agüero 2001).

La tradición de Valles Occidentales, MaitasChiribaya (ca. 800-1290 DC) corresponde a un tipo cerámico que tecnológica y decorativamente, se distancia de la alfarería altiplánica y, en cambio, presenta fuertes nexos con la de los Valles Occidentales del extremo sur de Perú, constituyendo la base de la cerámica local del PIT. Se caracteriza por pucos, jarros y cántaros de pastas arenosas a granulosas, con una estandarizada decoración de motivos en negro y blanco o crema, sobre fondo de revestimiento rojo (Espoueys et al. 1995; Uribe 1999).

Espoueys et al. (1995) al discutir la cronología de la cerámica del Valle de Azapa del periodo Medio mediante fechados de TL, coincide en términos generales con la secuencia planteada por Dauelsberg (1972/1973). Los fechados obtenidos de estilos que caracterizarían el periodo Medio Temprano, estaría dado por Charcollo, que marcaría un rango de 650 a 850 DC; el estilo Tiwanaku cubriría un rango de los 650 DC a 1270 DC y sería muy similar al estilo Cabuza que iría de los 500 a 1225 DC. El periodo Medio Tardío estaría representado por el estilo Maitas con un rango desde los 840 a 1290 DC y que a su vez sería contemporáneo con el estilo San Miguel Temprano que iría desde los 990 a 1030 DC y que representaría el comienzo del PIT en los valles de Arica. Para Espoueys et al. (1995) la población que manufacturó estos estilos tenían una economía agrícola y recolectora, además eran hábiles tejedores de fibras de lana y algodón.

Esta secuencia cronológica de estilos planteada por Dauelsberg (1985) y Espoueys et al. (1995) presenta sus primeros cambios con el trabajo de Focacci (1982) quien a partir de contextos funerarios fechados por $\mathrm{C} 14$, 
señala que la primera fase del periodo Medio estaría representada por una cerámica de fondo rojo decorada con motivos en negro correspondiendo al estilo Cabuza. Señala que los grupos que manufacturaron esta alfarería habrían sido agricultores y ganaderos provenientes de los Valles Occidentales precordilleranos. Sus espacios de asentamientos se habrían ubicado cerca de los recursos de agua, y con un amplio dominio visual. Señala que entre sus ceremonias hubo un culto al felino y un fuerte simbolismo en los rituales propiciatorios de fertilidad. A partir de los fechados obtenidos, reconoce que Tiwanaku habría tenido una débil penetración ocurrida entre el 900 al 1200 DC y lo caracterizarían las tumbas que tienen ofrendas cerámicas estilo Loreto Viejo. Esta presencia tardía de Tiwanaku habría coexistido con grupos que manufacturaban cerámicas Maitas y Chribaya que representaría la segunda fase del periodo Medio ${ }^{1}$

Por su parte Muñoz (2004), tomando como referencia las dos fases descritas por Focacci (1982) señala que la primera se vincularía al estilo Cabuza (600-1000 DC), que presenta similitudes en cuanto a la forma y estilo con la alfarería Tiwanaku periodo $\mathrm{V}^{2}$, esta similitud tal vez refleje la intención de reproducir el estilo altiplánico en los valles de Arica ${ }^{3}$. Hacia el 700 DC el estilo Cabuza habría coexistido con la cerámica estilo Maitas, que habría alcanzado un tiempo de ocupación de 500 años aproximadamente (700 al 1200 DC). Lo interesante del estilo Maitas es su decoración tricroma, estilo que difiere de los del área altiplánica meridional, teniendo más relación con otros estilos cerámicos del sur de Perú y la zona altiplánica oriental como: Chiribaya, Churajón, Allita Amaya, Mollo (Muñoz 1983).

Uribe (1999) al igual que los autores citados anteriormente señala que, durante el periodo Medio es evidente el predominio del estilo (tipo) Cabuza, al parecer este correspondería a un modelo altiplánico vinculado con Tiwanaku. Con este estilo hace su aparición la decoración pintada, en conjunto con la modelada. A pesar de su reducida presencia, el tipo Tiwanaku da cuenta que dicha entidad habría provocado un profundo impacto en las poblaciones del Valle de Azapa. Al referirse al estilo Maitas-Chiribaya, plantea al igual que Muñoz y Focacci (1985) un notable alejamiento de los patrones altiplánicos difundidos por Cabuza, la que dificulta relacionarlo con Tiwanaku.

En los últimos años, Korpisaari et al. (2014) y Korpisaari y Chacama (2015), a partir de fechados de $\mathrm{C} 14$, provenientes de cuatro cementerios del Valle de Azapa (Az-6, Az-71a, Az-141 y Az-143), señalan que la influencia Tiwanaku en este valle reflejaría procesos producidos por el colapso de la cultura altiplánica, más que un intento colonizador o de control indirecto llevado a cabo durante el Horizonte Medio ${ }^{4}$
Esta reflexión pues viene a confirmar estudios previos como los anteriormente citados y nos lleva a preguntarnos isi Tiwanaku no estuvo en Azapa durante el periodo Medio, quiénes fueron los actores sociales que marcaron presencia?

\section{La Influencia Cultural de Tiwanaku durante el Periodo Medio en el Valle de Azapa}

El poblamiento humano vinculado al periodo Medio en el Valle de Azapa ha sido el más estudiado en relación con el tema Tiwanaku, teniendo como antecedente los estudios sobre cronología y estilos definidos para la cerámica en su forma y estilo. Esta información ha sido fundamental para que, a partir de la década de los noventa se generara una discusión sobre la forma de operar desde el punto de vista político-económico de la cultura altiplánica en dicho valle. Chacama (2004), presenta dos interpretaciones confrontadas teórica y metodológicamente. La primera señala que el desarrollo de la agricultura habría sido consecuencia de la presencia de colonias y el predominio tecnológico de la influencia altiplánica en el modo de vida de las poblaciones del valle (Berenguer 1988, 2000; Berenguer y Dauelsberg 1989; Foccaci 1990; Goldstein 2006; Rivera 2002), mientras que la segunda resalta los antecedentes organizativos de los grupos locales como base para la estructura política y económica del valle durante el periodo Medio (Cassman 1997; Espoueys et al. 1995; Muñoz 2004; Uribe 1999; Uribe y Agüero 2001). En la primera interpretación, los contingentes coloniales procedentes del Altiplano se asentaron en aldeas contiguas a tierras con alto índice de producción, alrededor del 400 DC. Autores como Berenguer y Dauelsberg (1989) y Foccaci (1990) propusieron que los primeros asentamientos Tiwanaku fueron más pequeños que los asentamientos locales ya existentes, vinculados con la Fase Alto Ramírez y convivieron por un periodo de tiempo no determinado con los grupos locales. Según dichos autores, la aparición en algunos contextos funerarios de cerámica estilo Loreto Viejo, unido a una tradición textil propia del altiplano alrededor del $380 \mathrm{DC}$, fueron el reflejo de las primeras incursiones de colonias conformadas por pequeños grupos de élite, que con el tiempo llegaron a tener un estatus privilegiado frente a la población local.

Siguiendo esta propuesta, alrededor del 750 DC dos entidades culturales pueden ser identificadas poblando el Valle de Azapa: Cabuza y Maitas-Chiribaya que se diferenciaban por las características tecnológicas y estilísticas de su materialidad (Berenguer y Dauelsberg 1989; Goldstein 1995-1996, 2006). Sin embargo, estos planteamientos han brindado un panorama sesgado de la organización social de las poblaciones de los valles costeros debido a que están sustentados en análisis 
tipológicos de materiales hallados en contextos funerarios.

De manera distinta, trabajos más recientes han apelado a la existencia de un panorama más complejo durante este periodo, en el cual las poblaciones locales fueron agentes activos en la configuración de la estructura social de la época. Por ejemplo, los trabajos de análisis cerámico de Uribe (1999) señalan que, tanto Cabuza como Maitas, asimilan de manera distinta la influencia Tiwanaku. Para este autor, Cabuza llega a adoptar varios patrones estilísticos de Tiwanaku debido a que fue fuertemente impactado por esta cultura, mientras que Maitas presenta un patrón distinto, debido a que pudo haber reaccionado contra la imposición del poder ideológico de Tiwanaku, dando inicio a una cultura local que después derivaría en desarrollos políticos como la Cultura Arica. Asimismo, Uribe y Agüero (2001) señalan que los centros periféricos del Valle de Moquegua habrían tenido un mayor impacto en las poblaciones como el caso del Valle de Azapa, al convertirse en puentes de comunicación y dispersión cultural del núcleo altiplánico 5 . Ambos autores apelan a la generación de vínculos entre poblaciones de naturaleza variable, basados en los distintos intereses, tanto de las sociedades altiplánicas como de las locales. De manera complementaria, los estudios bioarqueológicos ayudaron a sustentar esta posición. Por ejemplo, los análisis de marcadores genéticos dentales realizados por Sutter (2006) señalan que la mayor parte de la población de Azapa presenta una continuidad biológica desde el período Arcaico hasta el Inka. Esta continuidad indicaría la existencia de una única población, que si bien a lo largo del tiempo recibió aportes de otras poblaciones, principalmente altiplánicas, estos no fueron suficientes para alterar en gran medida el patrón genético.

Recientes estudios de Muñoz y Gordillo (2016) acerca de la organización espacial del Valle de Azapa, señalan que la ocupación del valle durante el periodo Medio se distribuyó en los sectores medios, más allá del kilómetro 20 de la carretera principal de Azapa, concentrándose por sobre el plano aluvial riverino, cerca de las vertientes naturales de Las Riberas, Alto Ramírez y Saucache, indicando una marcada preferencia por las zonas con irrigación óptima para la agricultura (Goldstein 1995-1996, 2006; Muñoz 1995/1996; Muñoz y Focacci 1985; Muñoz y Zalaquett 2015; Rivera 1983). Esta forma de ocupar el espacio tiene una larga vigencia que se remonta desde los primeros agricultores, todo lo cual indica la importancia de las poblaciones locales en el contexto de definir los espacios de habitabilidad y producción agrícola en concordancia con el paisaje cultural.

Las propuestas planteadas en el párrafo anterior han iniciado un cuestionamiento a la naturaleza del orden social, político e ideológico con la cual Tiwanaku se había legitimado en las áreas extra-nucleares, enfatizando entre otros aspectos, la importancia y el rol activo que las periferias, en este caso las poblaciones azapeñas, habrían tenido en el contexto de la organización política y económica en los Valles Occidentales.

\section{Dataciones y Análisis Estadísticos del Período Medio en el Valle de Azapa}

Teniendo presente los distintos planteamientos sobre estilo y cronología, analizaremos las dataciones de C14 y TL obtenidas para el Valle de Azapa, durante el periodo Medio. Esta información se presenta en la Tabla 1 y se desglosa según la muestra, en una serie de categorías que incluye: sitio (sigla), tipo de sitio, estilo, período, fechado C14 y TL, no calibrado y fuente. La idea de integrar dataciones obtenidas a través de dos métodos de datación distintos, tiene como objetivo presentar un cuadro mayor de fechados relacionado con el periodo Medio. Además en la mayoría de los casos los fechados son coincidentes, lo cual permite enriquecer la discusión en relación a los objetivos planteados. Los antecedentes recopilados corresponden a 116 dataciones no calibradas obtenidas a través de información bibliográfica. Consisten en 60 fechados obtenidos por método de TL y 56 fechados por C14. Estos registros se acompañan de nuevas dataciones, las que en su mayoría provienen del cementerio Az-115, permitiendo una amplia discusión cronológica del periodo Medio.

El análisis estadístico se basó en estadígrafos descriptivos de tendencia central, de dispersión y de posición. Además, los datos se presentan como aplicaciones basados en gráficos y diagramas de caja y bigote mostrando datos de manera individual y comparativo entre los criterios escogidos, esto es: estilo de cerámica y referencia. Previo al análisis estadístico de los registros, estos se ordenaron conforme una máscara de ingreso de información que utilizó la hoja de cálculo Excel, de modo de adecuar una base de datos que fue analizada con el software de código abierto $\mathrm{R}$ 3.2.0 que generó la descripción estadística presentada.

Los resultados de este análisis se presentan en tres diagramas (Figuras 10, 11 y 12) de edades C14 por Estilo, TL por Estilo y de edades según métodos C14 y TL, de acuerdo con la información obtenida de las Tablas 2, 3 y 4.

\section{Resultados y Nuevas Dataciones}

Para dataciones C14 desagregadas por estilos se aprecia que Alto Ramírez III presenta una distribución alejada del resto de las fechas relativas a los otros estilos (Cabuza, Loreto Viejo, Maitas y Tiwanaku), donde prácticamente todas estas exhiben una mediana entre los 970 DC (Maitas) y 1066 DC (Loreto Viejo). 
Tabla 1. Dataciones del período Medio, a través del método de Termoluminiscencia (TL) y Carbono 14 (C14), Valle de Azapa y costa sur de Arica.

Datings of the Middle period, through the method of Thermoluminescence (TL) and Carbon 14 (C14), Azapa Valley and south coast of Arica.

\begin{tabular}{|c|c|c|c|c|c|c|c|}
\hline $\mathrm{N}^{\mathrm{o}}$ & Sigla & Tipo Sitio & Estilo/Tipo & Período & $\begin{array}{c}\text { Datación } \\
\text { C14 } \\
\text { (DC) }\end{array}$ & $\begin{array}{l}\text { Datación } \\
\text { TL } \\
\text { (DC) }\end{array}$ & Referencia \\
\hline 1 & $\mathrm{AZ}-143$ & Cementerio & Tiwanaku & Medio & - & $310 \pm 120$ & Schiappacasse et al. 1991 \\
\hline 2 & AZ - 71 & Piso habitacional & Alto Ramírez III & Medio & - & $470 \pm 200$ & Schiappacasse et al. 1991 \\
\hline 3 & AZ - 75 & Cementerio & Cabuza & Medio & - & $560 \pm 80$ & Schiappacasse et al. 1991 \\
\hline 4 & $\mathrm{AZ}-143$ & Cementerio & Cabuza & Medio & - & $755 \pm 120$ & Schiappacasse, et. al. 1991 \\
\hline 5 & AZ - 141 & Cementerio & Cabuza & Medio & - & $890 \pm 100$ & Schiappacasse et al. 1991 \\
\hline 6 & $\mathrm{AZ}-75$ & Cementerio & Cabuza & Medio & - & $760 \pm 80$ & Schiappacasse et al. 1991 \\
\hline 7 & AZ - 19 & Cementerio & Tiwanaku/Wari & Medio & - & $980 \pm 80$ & Schiappacasse et al. 1991 \\
\hline 8 & $\mathrm{AZ}-75$ & Cementerio & Maitas & Medio & - & $995 \pm 80$ & Schiappacasse et al. 1991 \\
\hline 9 & $\mathrm{AZ}-141$ & Cementerio & Tiwanaku & Medio & - & $1010 \pm 110$ & Schiappacasse et al. 1991 \\
\hline 10 & $A Z-143$ & Cementerio & Tiwanaku & Medio & - & $1020 \pm 100$ & Schiappacasse et al. 1991 \\
\hline 11 & AZ - 6 & Cementerio & Cabuza & Medio & - & $1020 \pm 100$ & Schiappacasse et al. 1991 \\
\hline 12 & AZ - 75 & Cementerio & Tiwanaku & Medio & - & $1070 \pm 100$ & Schiappacasse et al. 1991 \\
\hline 13 & $A Z-6$ & Cementerio & Cabuza & Medio & - & $1070 \pm 120$ & Schiappacasse et al. 1991 \\
\hline 14 & AZ - 115 & Cementerio & Alto Ramírez III & Medio & - & $340 \pm 170$ & Muñoz 1995/1996 \\
\hline 15 & $\mathrm{AZ}-70$ & Cementerio & Alto Ramírez III & Medio & - & $630 \pm 110$ & Espoueys et al. 1995 \\
\hline 16 & $\mathrm{AZ}-3$ & Cementerio & Charcollo & Medio & - & $720 \pm 100$ & Espoueys et al. 1995 \\
\hline 17 & $\mathrm{AZ}-70$ & Cementerio & Alto Ramírez III & Medio & - & $765 \pm 130$ & Espoueys et al. 1995 \\
\hline 18 & AZ - 3 & Cementerio & Charcollo & Medio & - & $830 \pm 90$ & Espoueys et al. 1995 \\
\hline 19 & AZ - 3 & Cementerio & Charcollo & Medio & - & $830 \pm 100$ & Espoueys et al. 1995 \\
\hline 20 & AZ - 103 & Cementerio & Charcollo & Medio & - & $850 \pm 90$ & Espoueys et al. 1995 \\
\hline 21 & $\mathrm{AZ}-3$ & Cementerio & Tiwanaku & Medio & - & $650 \pm 130$ & Espoueys et al. 1995 \\
\hline 22 & AZ - 3 & Cementerio & Tiwanaku & Medio & - & $720 \pm 130$ & Espoueys et al. 1995 \\
\hline 23 & $\mathrm{AZ}-3$ & Cementerio & Tiwanaku & Medio & - & $760 \pm 150$ & Espoueys et al. 1995 \\
\hline 24 & AZ - 103 & Cementerio & Tiwanaku & Medio & - & $785 \pm 110$ & Espoueys et al. 1995 \\
\hline 25 & AZ - 143 & Cementerio & Tiwanaku & Medio & - & $860 \pm 120$ & Espoueys et al. 1995 \\
\hline 26 & $\mathrm{AZ}-3$ & Cementerio & Tiwanaku & Medio & - & $865 \pm 100$ & Espoueys et al. 1995 \\
\hline 27 & AZ - 103 & Cementerio & Tiwanaku & Medio & - & $870 \pm 90$ & Espoueys et al. 1995 \\
\hline 28 & $A Z-103$ & Cementerio & Tiwanaku & Medio & - & $885 \pm 120$ & Espoueys et al. 1995 \\
\hline 29 & AZ - 3 & Cementerio & Tiwanaku & Medio & - & $890 \pm 100$ & Espoueys et al. 1995 \\
\hline 30 & AZ - 3 & Cementerio & Tiwanaku & Medio & - & $915 \pm 110$ & Espoueys et al. 1995 \\
\hline 31 & AZ - 3 & Cementerio & Tiwanaku & Medio & - & $920 \pm 100$ & Espoueys et al. 1995 \\
\hline 32 & AZ - 3 & Cementerio & Tiwanaku & Medio & - & $950 \pm 130$ & Espoueys et al. 1995 \\
\hline 33 & $\mathrm{AZ}-1$ & Cementerio & Tiwanaku & Medio & - & $955 \pm 90$ & Espoueys et al. 1995 \\
\hline 34 & AZ - 3 & Cementerio & Tiwanaku & Medio & - & $1060 \pm 90$ & Espoueys et al. 1995 \\
\hline 35 & AZ - 3 & Cementerio & Tiwanaku & Medio & - & $1085 \pm 90$ & Espoueys et al. 1995 \\
\hline 36 & AZ - 103 & Cementerio & Tiwanaku & Medio & - & $1175 \pm 90$ & Espoueys et al. 1995 \\
\hline 37 & $A Z-103$ & Cementerio & Tiwanaku & Medio & - & $1270 \pm 80$ & Espoueys et al. 1995 \\
\hline 38 & $\mathrm{AZ}-3$ & Cementerio & Cabuza & Medio & - & $500 \pm 140$ & Espoueys et al. 1995 \\
\hline 39 & $A Z-13$ & Cementerio & Cabuza & Medio & - & $775 \pm 120$ & Espoueys et al. 1995 \\
\hline 40 & AZ - 3 & Cementerio & Cabuza & Medio & - & $805 \pm 100$ & Espoueys et al. 1995 \\
\hline 41 & $\mathrm{AZ}-3$ & Cementerio & Cabuza & Medio & - & $860 \pm 80$ & Espoueys et al. 1995 \\
\hline 42 & $\mathrm{AZ}-3$ & Cementerio & Cabuza & Medio & - & $865 \pm 80$ & Espoueys et al. 1995 \\
\hline 43 & $\mathrm{AZ}-3$ & Cementerio & Cabuza & Medio & - & $890 \pm 100$ & Espoueys et al. 1995 \\
\hline 44 & $\mathrm{AZ}-3$ & Cementerio & Cabuza & Medio & - & $935 \pm 80$ & Espoueys et al. 1995 \\
\hline 45 & AZ - 3 & Cementerio & Cabuza & Medio & - & $940 \pm 50$ & Espoueys et al. 1995 \\
\hline 46 & $\mathrm{AZ}-3$ & Cementerio & Cabuza & Medio & - & $970 \pm 80$ & Espoueys et al. 1995 \\
\hline 47 & $A Z-3$ & Cementerio & Cabuza & Medio & - & $1070 \pm 90$ & Espoueys et al. 1995 \\
\hline 48 & $A Z-103$ & Cementerio & Cabuza & Medio & - & $1120 \pm 90$ & Espoueys et al. 1995 \\
\hline 49 & AZ - 3 & Cementerio & Cabuza & Medio & - & $1195 \pm 80$ & Espoueys et al. 1995 \\
\hline 50 & AZ - 103 & Cementerio & Cabuza & Medio & - & $1225 \pm 70$ & Espoueys et al. 1995 \\
\hline 51 & $\mathrm{AZ}-3$ & Cementerio & Cabuza/Sobraya & Medio & - & $675 \pm 85$ & Espoueys et al. 1995 \\
\hline 52 & $\mathrm{AZ}-3$ & Cementerio & Maitas/Chiribaya & Medio & - & $840 \pm 110$ & Espoueys et al. 1995 \\
\hline 53 & AZ - 79 & Cementerio & Maitas/Chiribaya & Medio & - & $890 \pm 100$ & Espoueys et al. 1995 \\
\hline 54 & AZ - 3 & Cementerio & Maitas/Chiribaya & Medio & - & $950 \pm 110$ & Espoueys et al. 1995 \\
\hline 55 & AZ - 3 & Cementerio & Maitas/Chiribaya & Medio & - & $1120 \pm 90$ & Espoueys et al. 1995 \\
\hline
\end{tabular}


Continuación Tabla 1.

\begin{tabular}{|c|c|c|c|c|c|c|c|}
\hline $\mathrm{N}^{\mathrm{o}}$ & Sigla & Tipo Sitio & Estilo/Tipo & Período & $\begin{array}{c}\text { Datación } \\
\text { C14 } \\
\text { (DC) }\end{array}$ & $\begin{array}{c}\text { Datación } \\
\text { TL } \\
\text { (DC) }\end{array}$ & Referencia \\
\hline 56 & AZ - 8 & Cementerio & Maitas/Chiribaya & Medio & - & $1160 \pm 80$ & Espoueys et al. 1995 \\
\hline 57 & $\mathrm{AZ}-75$ & Cementerio & Maitas/Chiribaya & Medio & - & $1215 \pm 60$ & Espoueys et al. 1995 \\
\hline 58 & AZ - 105 & Cementerio & Maitas/Chiribaya & Medio & - & $1260 \pm 70$ & Espoueys et al. 1995 \\
\hline 59 & AZ - 3 & Cementerio & Maitas/Chiribaya & Medio & - & $1260 \pm 60$ & Espoueys et al. 1995 \\
\hline 60 & $\mathrm{AZ}-8$ & Cementerio & Maitas/Chiribaya & Medio & - & $1290 \pm 70$ & Espoueys et al. 1995 \\
\hline 61 & AZ - 6 & Cementerio & Cabuza/Sobraya & Medio & $380 \pm 65$ & - & Rivera 1980; Focacci 1982 \\
\hline 62 & AZ - 6 & Cementerio & Maitas/Chiribaya & Medio & $730 \pm 80$ & - & Rivera 1980; Focacci 1982 \\
\hline 63 & AZ - 6 & Cementerio & Maitas/Chiribaya & Medio & $1235 \pm 130$ & - & Focacci 1982 \\
\hline 64 & AZ - 6 & Cementerio & Maitas/Chiribaya & Medio & $1040 \pm 145$ & - & Focacci 1982 \\
\hline 65 & $\mathrm{AZ}-11$ & Habitacional & Cabuza/Sobraya & Medio & $590 \pm 80$ & - & Focacci 1982 \\
\hline 66 & PLM - 9 & Cementerio & Maytas/San Miguel & Medio & $895 \pm 80$ & - & Focacci 1982 \\
\hline 67 & AZ - 71 & Cementerio & Maitas/Chiribaya & Medio & $1185 \pm 75$ & - & Focacci 1982 \\
\hline 68 & $A Z-71$ & Cementerio & Maitas/Chiribaya & Medio & $1255 \pm 75$ & - & Focacci 1982 \\
\hline 69 & $\mathrm{AZ}-71$ & Cementerio & Loreto Viejo & Medio & $956 \pm 75$ & - & Focacci 1982 \\
\hline 70 & AZ - 71 & Cementerio & Loreto Viejo & Medio & $1176 \pm 75$ & - & Focacci 1982 \\
\hline 71 & $\mathrm{AZ}-71$ & Cementerio & Maitas & Medio & $880 \pm 80$ & - & Focacci y Muñoz 1983 \\
\hline 72 & AZ - 71 & Cementerio & Maitas & Medio & $970 \pm 80$ & - & Focacci y Muñoz 1983 \\
\hline 73 & AZ - 71 & Cementerio & Maitas & Medio & $950 \pm 80$ & - & Focacci y Muñoz 1983 \\
\hline 74 & AZ - 140 & Cementerio & Maitas & Medio & $766 \pm 80$ & - & Focacci y Muñoz 1983 \\
\hline 75 & AZ - 140 & Cementerio & Maitas & Medio & $970 \pm 80$ & - & Focacci y Muñoz 1983 \\
\hline 76 & La Cap- 4 & Deposito & Maitas & Medio & $700 \pm 100$ & - & Muñoz 1983 \\
\hline 77 & AZ - 11 & Habitacional & Maitas & Medio & $790 \pm 80$ & - & Muñoz 1983 \\
\hline 78 & $\mathrm{AZ}-11$ & Habitacional & Maitas & Medio & $860 \pm 80$ & - & Muñoz 1983 \\
\hline 79 & AZ - 11 & Habitacional & Maitas & Medio & $980 \pm 80$ & - & Muñoz 1983 \\
\hline 80 & AZ - 11 & Habitacional & Maitas & Medio & $860 \pm 80$ & - & Muñoz 1983 \\
\hline 81 & $\mathrm{AZ}-11$ & Habitacional & Maitas & Medio & $830 \pm 80$ & - & Muñoz 1983 \\
\hline 82 & $A Z-83$ & Habitacional & Alto Ramírez III & Medio & $560 \pm 110$ & - & Rivera 1980 \\
\hline 83 & AZ - 83 & Habitacional & Alto Ramírez III & Medio & $760 \pm 70$ & - & Rivera 1980 \\
\hline 84 & AZ - 75 & Cementerio & Alto Ramírez III & Medio & $325 \pm 80$ & - & Muñoz 1995/1996 \\
\hline 85 & AZ - 75 & Cementerio & Alto Ramírez III & Medio & $670 \pm 265$ & - & Muñoz 1995/1996 \\
\hline 86 & PLM - 9 & Cementerio & Alto Ramírez III & Medio & $230 \pm 60$ & - & Cassman 1997 \\
\hline 87 & PLM - 9 & Cementerio & Alto Ramírez III & Medio & $810 \pm 50$ & - & Cassman 1997 \\
\hline 88 & AZ - 71 & Cementerio & Alto Ramírez III & Medio & $840 \pm 80$ & - & Cassman 1997 \\
\hline 89 & $\mathrm{AZ}-140$ & Cementerio & Alto Ramírez III & Medio & $990 \pm 70$ & - & Cassman 1997 \\
\hline 90 & $\mathrm{AZ}-71$ & Cementerio & Alto Ramírez III & Medio & $990 \pm 70$ & - & Cassman 1997 \\
\hline 91 & AZ - 71 & Cementerio & Alto Ramírez III & Medio & $1000 \pm 60$ & - & Cassman 1997 \\
\hline 92 & AZ - 71 & Cementerio & Alto Ramírez III & Medio & $1000 \pm 50$ & - & Cassman 1997 \\
\hline 93 & AZ - 71 & Cementerio & Alto Ramírez III & Medio & $1010 \pm 50$ & - & Cassman 1997 \\
\hline 94 & AZ - 71 & Cementerio & Alto Ramírez III & Medio & $1030 \pm 40$ & - & Cassman 1997 \\
\hline 95 & AZ - 71 & Cementerio & Alto Ramírez III & Medio & $1040 \pm 40$ & - & Cassman 1997 \\
\hline 96 & $\mathrm{AZ}-71$ & Cementerio & Alto Ramírez III & Medio & $1050 \pm 70$ & - & Cassman 1997 \\
\hline 97 & $\mathrm{AZ}-140$ & Cementerio & Alto Ramírez III & Medio & $1070 \pm 60$ & - & Cassman 1997 \\
\hline 98 & $\mathrm{AZ}-71$ & Cementerio & Alto Ramírez III & Medio & $1070 \pm 50$ & - & Cassman 1997 \\
\hline 99 & PLM - 9 & Cementerio & Alto Ramírez III & Medio & $1070 \pm 60$ & - & Cassman 1997 \\
\hline 100 & $\mathrm{AZ}-140$ & Cementerio & Alto Ramírez III & Medio & $1080 \pm 70$ & - & Cassman 1997 \\
\hline 101 & $\mathrm{AZ}-71$ & Cementerio & Alto Ramírez III & Medio & $1090 \pm 70$ & - & Cassman 1997 \\
\hline 102 & PLM - 9 & Cementerio & Alto Ramírez III & Medio & $1120 \pm 50$ & - & Cassman 1997 \\
\hline 103 & $A Z-6$ & Cementerio & Maitas/Chiribaya & Medio & $1046 \pm 29$ & - & Korpisaari et al. 2014 \\
\hline 104 & $\mathrm{AZ}-143$ & Cementerio & Tiwanaku & Medio & $1161 \pm 32$ & - & Korpisaari et al. 2014 \\
\hline 105 & $\mathrm{AZ}-143$ & Cementerio & Tiwanaku & Medio & $893 \pm 28$ & - & Korpisaari et al. 2014 \\
\hline 106 & AZ - 6 & Cementerio & Cabuza & Medio & $1046 \pm 29$ & - & Korpisaari et al. 2014 \\
\hline 107 & AZ - 6 & Cementerio & Cabuza & Medio & $1222 \pm 28$ & - & Korpisaari et al. 2014 \\
\hline 108 & AZ - 6 & Cementerio & Cabuza & Medio & $1158 \pm 28$ & - & Korpisaari et al. 2014 \\
\hline 109 & $A Z-6$ & Cementerio & Cabuza & Medio & $1152 \pm 28$ & - & Korpisaari et al. 2014 \\
\hline 110 & $A Z-6$ & Cementerio & Cabuza & Medio & $1046 \pm 28$ & - & Korpisaari et al. 2014 \\
\hline 111 & $A Z-6$ & Cementerio & Cabuza & Medio & $1160 \pm 29$ & - & Korpisaari et al. 2014 \\
\hline 112 & $A Z-71 a$ & Cementerio & Maitas/Chiribaya & Medio & $1228 \pm 35$ & - & Korpisaari et al. 2014 \\
\hline 113 & $A Z-71 a$ & Cementerio & Cabuza & Medio & $1299 \pm 37$ & - & Korpisaari et al. 2014 \\
\hline 114 & $\mathrm{AZ}-71 \mathrm{a}$ & Cementerio & Cabuza & Medio & $1051 \pm 34$ & - & Korpisaari et al. 2014 \\
\hline 115 & AZ - 141 & Cementerio & Cabuza & Medio & $1204 \pm 34$ & - & Korpisaari et al. 2014 \\
\hline 116 & $\mathrm{AZ}-141$ & Cementerio & Cabuza & Medio & $1044 \pm 35$ & - & Korpisaari et al. 2014 \\
\hline
\end{tabular}




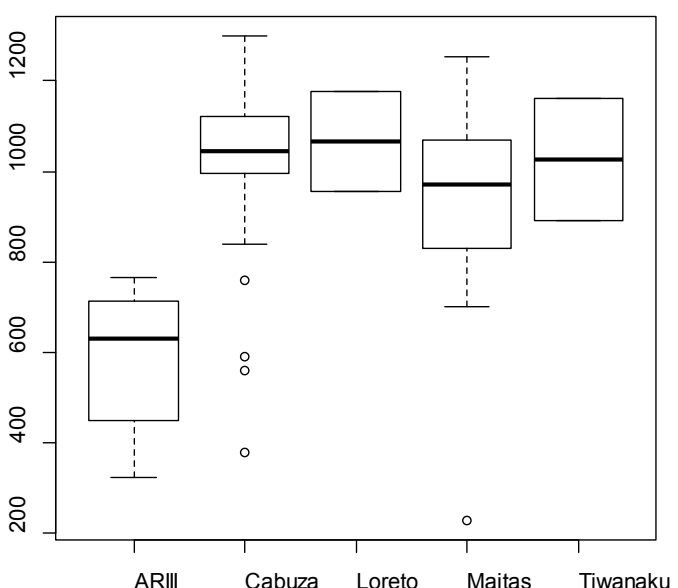

Figura 10. Diagrama Box Plot de edades C14 por estilo. Box Plot diagram of C14 ages by style.

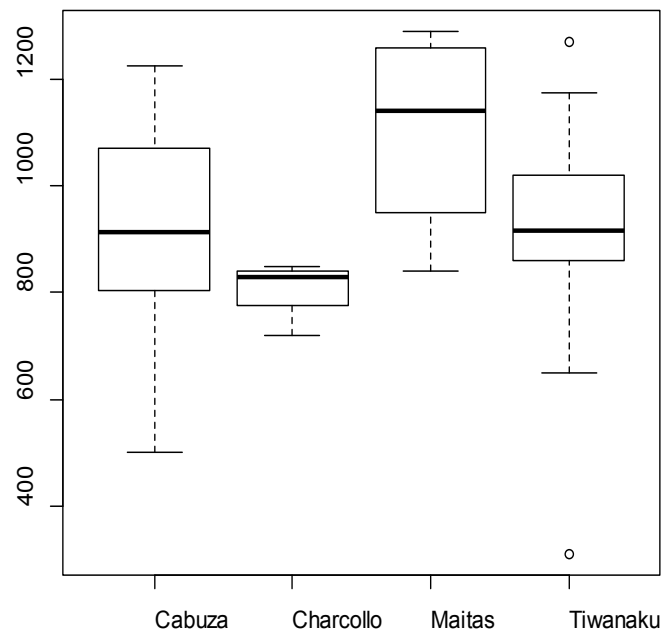

Figura 11. Diagrama Box Plot de edades TL por estilo. Box Plot diagram of TL ages by style.

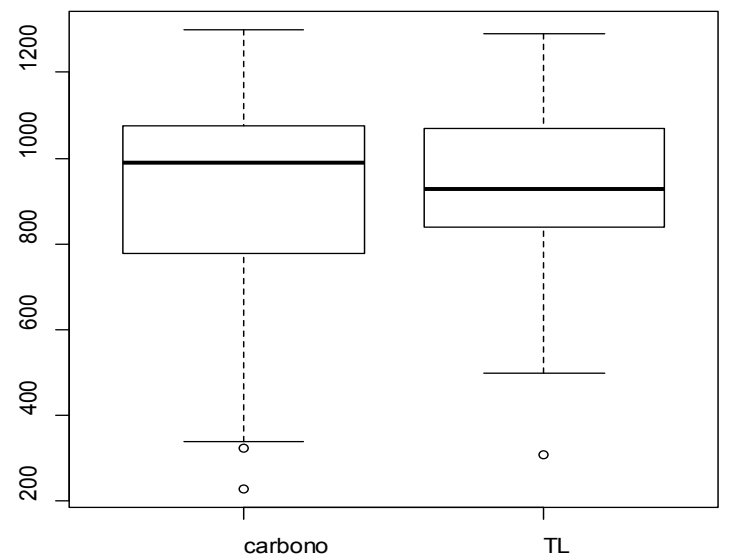

Figura 12. Diagrama Box Plot de edades según métodos C14 y TL.

Box Plot diagram of ages according to C14 and TL methods.

Tabla 2. Descriptivo por tipo de estilo C14. Descriptive by $\mathrm{C} 14$ style type.

\begin{tabular}{lccccccc}
\hline \multicolumn{7}{c}{ C 14 (años) } \\
\hline \multicolumn{1}{c}{ Estilo/Estadístico } & Min. & Median & Mean & Max. & Sd & R & cv \\
\hline Alto Ramírez III & 325 & 630 & 578,6 & 765 & 182,68 & 440 & 31,57 \\
Cabuza & 380 & 1045 & 991,3 & 1299 & 219,53 & 919 & 22,14 \\
Loreto Viejo & 956 & 1066 & 1066 & 1176 & 155,56 & 220 & 14,59 \\
Maitas & 230 & 970 & 939,8 & 1255 & 217,65 & 1025 & 23,16 \\
Tiwanaku & 893 & 1027 & 1027 & 1094 & 189,5 & 201 & 18,45 \\
\hline
\end{tabular}


Tabla 3. Descriptivo por tipo de estilo TL.

Descriptive by TL style type.

\begin{tabular}{|c|c|c|c|c|c|c|c|}
\hline \multicolumn{8}{|c|}{ TL (años) } \\
\hline Estilo/Estadístico & Min. & Median & Mean & Max. & $\mathrm{Sd}$ & $\mathrm{R}$ & $\mathrm{cv}$ \\
\hline Cabuza & 500 & 912,5 & 920 & 1225 & 183,6 & 725 & 19,95 \\
\hline Charcollo & 720 & 830 & 807,5 & 850 & 59,09 & 130 & 7,31 \\
\hline Maitas & 840 & 1140 & 1098 & 1290 & 166,57 & 450 & 15,17 \\
\hline Tiwanaku & 310 & 917,5 & 909,3 & 1270 & 197,39 & 960 & 21,7 \\
\hline
\end{tabular}

Tabla 4. Descriptivos según métodos C14 y TL. Descriptive according to C14 and TL methods.

\begin{tabular}{lccccccc}
\hline \multicolumn{7}{c}{ TL (años) } \\
\hline \multicolumn{1}{c}{ Método/Estadístico } & Min. & Median & Mean & Max. & sd & R & cv \\
\hline Termoluminiscencia & 310 & 927,5 & 940,3 & 1290 & 194,22 & 980 & 20,65 \\
Carbono 14 & 230 & 990 & 925,4 & 1299 & 244,16 & 1069 & 26,38 \\
Ambos & 230 & 955,5 & 932,4 & 1299 & 221,08 & 1069 & 23,71 \\
\hline
\end{tabular}

Esta situación se debería a que Alto Ramírez III es una cerámica vinculada cronológicamente con el periodo Formativo Tardío y por lo tanto coincide con la fase temprana del periodo Medio. Entre otros aspectos no presenta decoración; sus formas y pastas se relacionan más bien con la cerámica formativa vinculada a la fase Alto Ramírez (Espoueys et al.1995; Muñoz 2004; Rivera 1980). Alto Ramírez III, exhibe una mediana de $630 \mathrm{DC}$, en cierta medida los datos son confiables ya que los coeficientes de variabilidad son aceptables (máximo 32\%). Por otra parte, el mayor rango lo exhibe el estilo Maitas con un periodo aproximado de 500 años; el más estrecho lo constituye el estilo Tiwanaku con solo 200 años.

Los fechados por TL, exhiben menor variedad de estilos (Cabuza, Charcollo, Maitas y Tiwanaku), en ellos se observan medianas de 830 - 1140 DC, años con coeficiente de variabilidad (CV). Al comparar ambos métodos, Cabuza TL arroja una mediana inferior a los del método del C14, un mínimo mayor y un máximo menor, por lo tanto, Cabuza C14, contiene a Cabuza TL. Para el caso Maitas se exhibe una mediana mayor en el caso de $\mathrm{C} 14$ y nuevamente ambos extremos se pueden anidar (insertar) en Maitas C14; finalmente Tiwanaku exhibe menores mediana y máximo, escapándose el mínimo que es inferior al Tiwanaku C14 se concluye así que para el caso de estos tres estilos en términos generales los rangos mostrados por C14 son más amplios que los de TL a excepción de Tiwanaku donde los rangos mostrados por TL son mucho más amplios que los rangos C14.

En términos generales (fechados obtenidos por el método TL, y C14) se observó que todos los años agregados mediante $\mathrm{C} 14$ tienen ligera amplitud sobre los de TL (1069 DC y 980 DC, respectivamente). Por lo tanto, se puede concluir que ambos métodos utilizados para datar indistintamente del periodo Medio, presentan ligeras variaciones de tiempo, según sea el criterio de desagregación utilizado ${ }^{6}$.

En síntesis, descriptivamente no se aprecia diferencia práctica entre ambos métodos, si potencialmente se realizara una prueba de hipótesis los más probable sería no encontrar diferencias significativas con alguna confiabilidad entre ambos métodos.

Recientemente, en el marco de proyecto FONDECYT 1130249 se muestreó el cementerio Az115 , donde se obtuvieron ocho dataciones $\mathrm{C} 14$, cuya primera fecha obtenida por TL (Muñoz 1995/1996) había arrojado una datación de 340 DC. Esta información nos alentó a datar nuevamente dicho cementerio con la idea de obtener nuevos fechados que se situaran dentro de los primeros seis siglos de la Era Cristiana, información fundamental para conocer los inicios y desarrollo del periodo Medio en el contexto de quiénes fueron las poblaciones que constituyeron dicho periodo y qué relación habrían tenido con las poblaciones Alto Ramírez que los antecedieron.

Complementa este nuevo conjunto de dataciones (Tabla 5) un fechado obtenido del cementerio Az-145, conocida como la waka de Cerro Blanco y una datación registrada del cementerio Az-143, ambos sitios con presencia de cerámica estilos Cabuza y Tiwanaku.

\section{Discusión en torno a la Cronología del periodo Medio a partir de las fechas de Az-115}

Con las fechas obtenidas del cementerio Az-115 se ha complementado la información cronológica para la secuencia arqueológica del valle de Azapa. En la actualidad contamos con 14 fechados que sitúan un proceso cultural que va desde comienzos de la Era Cristiana al 700 DC. 
Tabla 5. Dataciones C14, sitio Az-115, Az-143 y Az-145.

C14 datings, Az-115, Az-143 and Az-145 sites.

\begin{tabular}{|c|c|c|c|c|c|c|}
\hline $\begin{array}{l}\text { Código } \\
\text { muestra }\end{array}$ & Sitio & $14 \mathrm{C}$ años AP & $\mathrm{Cal} 2 \sigma \mathrm{AC}$ & $\delta 13 \mathrm{C}$ & Material & $\mathrm{N}^{\circ}$ laboratorio \\
\hline 24652 & $\begin{array}{l}\text { Az-115 tumba } \\
145 \text {, cuadricula } \mathrm{J} 2\end{array}$ & $1830 \pm 30$ & $\begin{array}{l}\text { Cal AD } 140 \text { to } 180 \text { (Cal BP } \\
1810 \text { to } 1770) \text { and Cal AD } \\
200 \text { to } 255(\mathrm{Cal} \mathrm{BP} 1750 \text { to } \\
1695) \text { and Cal AD } 285 \text { to } 340 \\
\text { (Cal BP } 1665 \text { to } 1610)\end{array}$ & NA & Tejido blando & BETA 394146 \\
\hline 24652 & $\begin{array}{l}\text { Az-115, tumba } \\
\text { 127, cuadricula J5 }\end{array}$ & $1760 \pm 30$ & $\begin{array}{c}\mathrm{Cal} \mathrm{AD} 245 \text { to } 390(\mathrm{Cal} \mathrm{BP} \\
1705 \text { to } 1560) \text { and } \mathrm{Cal} \mathrm{AD} 400 \\
\text { to } 405(\mathrm{Cal} \mathrm{BP} 1550 \text { to } 1545)\end{array}$ & $-21,7 \mathrm{o} / \mathrm{oo}$ & Tejido blando & BETA 394144 \\
\hline 24652 & $\begin{array}{l}\text { Az-115, tumba } \\
124, \text { cuadricula } \\
13-14\end{array}$ & $1800 \pm 30$ & $\begin{array}{c}\mathrm{Cal} \mathrm{AD} 215 \text { to } 360(\mathrm{Cal} \mathrm{BP} \\
1735 \text { to } 1590)\end{array}$ & NA & Tejido blando & BETA 394143 \\
\hline 24652 & $\begin{array}{l}\text { Az-115, tumba } 30, \\
\text { cuadricula L-5 }\end{array}$ & $1530 \pm 30$ & $\begin{array}{c}\mathrm{Cal} \mathrm{AD} 535 \text { to } 640(\mathrm{Cal} \mathrm{BP} \\
1415 \text { to } 1310)\end{array}$ & $-20,3 \mathrm{o} / \mathrm{oo}$ & Fibra vegetal & BETA 394141 \\
\hline 24652 & $\begin{array}{l}\text { Az- } 115, \text { tumba } \\
\text { 3, cuadricula S/ } \\
\text { inf., cuerpo de } \\
\quad \text { individuo }\end{array}$ & $1360 \pm 30$ & $\begin{array}{c}\mathrm{Cal} \mathrm{AD} 650 \text { to } 770(\mathrm{Cal} \mathrm{BP} \\
1300 \text { to } 1180)\end{array}$ & $-17,8 \mathrm{o} / \mathrm{oo}$ & Tejido blando & BETA 394140 \\
\hline 31178 & $\begin{array}{l}\text { Az-115 Tumba } \\
211, \text { Trinchera } 1 \\
\text { Contexto } 14\end{array}$ & $1790 \pm 30$ & $\begin{array}{c}\mathrm{Cal} \mathrm{AD} 225 \text { to } 365(\mathrm{Cal} \mathrm{BP} \\
1725 \text { to } 1585)\end{array}$ & $-13,5 \mathrm{o} / \mathrm{oo}$ & Tejido blando & BETA 412336 \\
\hline
\end{tabular}

Las dataciones obtenidas de los entierros de Az115 arrojaron fechas que van desde los 120 al 590 DC; sin embargo, las fechas calibradas alcanzan un rango mayor que iría desde los 100 al 700 DC. En este rango de tiempo no observamos un tipo de entierro más temprano que otro; por ejemplo, los entierros en cistas de los cuales se obtuvieron cuatro fechados, van desde los 150 a 540 DC. Por otro lado, las dataciones provenientes de entierros en urnas da una fecha de 440 DC, a diferencia de los cuerpos enfardados que van desde el 120 a 590 DC. Podemos concluir que las poblaciones enterradas en Az-115 tuvieron tres tipos de enterratorios, de los cuales dos son similares al patrón de entierro que caracterizan el periodo Formativo a lo menos 500 AC. Lo nuevo en relación con el periodo Formativo son los entierros en urnas confeccionadas en fibra vegetal y las edificaciones abovedadas, utilizando maderos para proteger el cuerpo (Muñoz 2017).

Los entierros de Az-115 serían contemporáneos con los entierros fechados por Muñoz (1995/1996) excavados en el cementerio de Az-75. En ellos, observamos un patrón de entierro similar y un ajuar con ausencia de cerámica pintada, y uno que otro rasgo con iconografía zoomorfa y geométrica. Ambos grupos, Az-115 y Az-75, estarían más bien relacionados a una tradición local en cuanto a la forma de enterrarse, y se remontaría al periodo Formativo tanto en la costa como en los valles, poblaciones que habrían sido responsables de agriculturizar el valle y de conformar los cimientos de una mayor complejidad social que se expresa más tardíamente en la fase Maitas-Chiribaya.

De las dataciones de $\mathrm{C} 14$ obtenidas del cementerio Az-115, sumadas a las de Az-75 (Muñoz 1995/1996) y Az-83 (Rivera 1987), se desprenden tres comentarios. En primer lugar, si consideramos las fechas más tardías de estos cementerios (400-600 DC), nos cabe la pregunta ¿fueron estas poblaciones las que iniciaron la organización agraria del valle culminando con la planificación y edificación del poblado de San Lorenzo?, para lograr este objetivo ambas poblaciones habrían logrado consolidar una economía agrícola 
complementada con recursos de caza, pesca y recolección marina y terrestre. Se asentaron en una franja territorial estratégica, ubicada en el sector medio del Valle de Azapa, donde se hallaban los mayores recursos hídricos. En segundo lugar, estas poblaciones a partir del $700 \mathrm{DC}$ al parecer lograron cohesionar una unidad social, administrativa, política y económica como lo plantean Muñoz y Peña (2018), lo cual permitió la planificación de la aldea de San Lorenzo (Az-11), el asentamiento más emblemático del desarrollo agrícola costero en los valles de Arica. En tercer lugar, desde el punto de vista de la alfarería, en Az-115 no hay cerámica decorada, los entierros presentan un patrón local, y lo novedoso es el trabajo en miniaturas en el arte textil, confeccionadas en fibra vegetal y lana. Estas poblaciones desde el punto de vista de la tecnología serían continuadoras de las poblaciones Alto Ramírez, cuya tradición textil está presente desde antes de la Era Cristiana (Ulloa 1982).

Los fechados de Az-115 y Az-75 son referencias cronológicas importantes para definir el primer momento (o fase) del periodo Medio, información desconocida anteriormente. Las fechas que hacían referencia a esta primera fase eran escasas y complejas desde el punto de vista de las muestras fechadas. Así tenemos, que la muestra de restos de mandioca de Az-6, dio una fecha de 360 DC (Rivera 1980). Por su parte, la muestra de músculo tomada de un cuerpo ubicado en el perímetro del poblado de San Lorenzo (Az-11), según Focacci (1982) corresponde a un entierro disturbado con ausencia de ofrendas. Por otro lado, resulta compleja la datación de los fragmentos cerámicos fechados por el método TL de los sitios Az-3 y Az-147 (400 al 600 DC) en el sentido que, la fecha de Az-3, está dentro de un rango de 50 muestras datadas, todas con fechas tardías, lo cual resulta ser una antecedente cronológico bastante particular y aislado, lo mismo que Az-147, las que podrían ser fechas aberrantes. Finalmente, los fechados de las muestras tomadas de los entierros de Az-145 y Az-143 se ubican dentro del rango de tiempo de fechas asociadas con cerámica estilos Cabuza y Tiwanaku que se sitúan entre los 900 DC al 1000 DC, marcando la fecha más probable de la transición al periodo Intermedio Tardío.

\section{Conclusiones}

\section{Estilos cerámicos y poblamiento humano en Azapa a partir del ordenamiento de los fechados}

La presencia de cerámica decorada en el Valle de Azapa parece ser más tardía de lo que se ha propuesto. Más bien se relacionaría a una segunda fase de agricultores, donde el proceso agrícola estaría consolidado con cultivos como maíz, ají, calabazas, camote, papas, jíquima, etc. (Muñoz 2014; Muñoz et al.
2016). Este fuerte desarrollo de la actividad agrícola, habría sido consecuencia de la canalización de las aguas de vertientes, lo que permitió regar amplios terrenos donde se sembraron variedades de cultivos, logrando una producción permanente. Desde el punto de vista de la organización de los asentamientos, construyeron entre otras obras arquitectónicas el emplazamiento de San Lorenzo, que pasó a ser con el tiempo una aldea ceremonial donde se habrían dado las relaciones de intercambio más marcadas. En esta aldea habrían convergido los senderos caravaneros, lo que lo habría convertido en un espacio de múltiples relaciones sociales y económicas y que, de alguna manera, se ve reflejado en los motivos que presenta el arte rupestre (petroglifos) donde se resaltan escenas de la vida cotidiana.

En el cementerio Az-75 donde se enterraron las poblaciones que habitaron San Lorenzo, se han encontrado una serie de ofrendas que al parecer fueron traídas de ambientes lejanos: mono (Aluatta seniculos), plumas de aves tropicales, maderas de chonta, metales, quinoa, cerámicas decoradas, etc., lo que sugiere un flujo de personas que habrían traído bienes y productos lo cual permitió que se generara un intercambio con las poblaciones del valle.

Es en este contexto, de movilidad y diversidad cultural donde se manifiesta la presencia de la influencia de Tiwanaku, los fechados en su gran mayoría resultan ser tardíos (900 al 1200 DC) en relación con el periodo Medio. Respecto a estos fechados en la mayoría de los casos, lo que se trató de datar, fueron muestras que se asociaran con la presencia de la cultura altiplánica (Tiwanaku) en el Valle de Azapa, con el propósito de establecer en qué momento de la historia se habría producido dicha relación cultural. Por lo tanto, las muestras de cerámica consideradas para el análisis por TL tuvieron como objetivo buscar formas e iconos que se asemejaran a dicha cultura. En el caso de las muestras por C14, tomadas por Rivera (1980) y Focacci (1982), estas correspondieron a fibras de músculo y pelo humano y en otros casos, a restos de productos agrícolas; en ambos casos, presentan como novedad el haber hallado en su contexto mortuorio piezas de cerámica que asemejaran al estilo altiplánico. En el caso de los fechados publicados en Korpisaari et al. (2014), se tomaron princpalmente muestras sacadas de recipientes de cestería hallados en tumbas, así como fibras textiles, vegetales y madera (Tabla 1; Korpisaari et al. 2014).

Los resultados de ambas propuestas TL y C14 apuntaron a conocer cronológicamente los inicios y desarrollo de la influencia altiplánica en Azapa, Arica, sin embargo las muestras datadas como se señaló anteriormente dieron fechas tardías, dejando una gran incógnita sobre quienes habrían ocupado el valle los primeros ocho siglos de la Era Cristiana.

Las escasas dataciones que registran las piezas Tiwanaku más tempranas del 850 al 900 DC en el Valle de Azapa, corresponden a objetos aislados donde no se 
aprecia intencionalidad por el cambio en el contexto de la estructura ideológica de las comunidades del Valle de Azapa. Por otro lado, la presencia tardía de Tiwanaku al parecer se habría dado en un contexto de cambio cuando las poblaciones locales se hallaban en la búsqueda de una identidad regional, por lo tanto, las poblaciones que interactuaron o integraron bienes con rasgos Tiwanaku en su acervo cultural, fueron más bien gente que estaba viviendo aires de cambio de naturaleza regional, que en nuestro caso se materializaría con la Cultura Arica. Esta hipótesis nos lleva a la pregunta ¿qué rol pudo haber tenido la presencia de piezas y objetos Tiwanaku en Azapa? Por los nuevos datos cronológicos y estudio de contextos, pareciera ser más bien materiales que habrían llegado e imitados por artesanos del valle. Su difusión en el contexto de las familias azapeñas habría sido percibido como una moda, rasgos que habrían ayudado a que se cimentara una nueva identidad en las poblaciones de valles a partir de nuevas ideas y compromisos que fueron adquiriendo las poblaciones locales, sin romper con una tradición cultural milenaria propia de estos valles.

Al margen de como pudo haber interactuado Tiwanaku en Azapa, lo concreto es que en la segunda fase del periodo Medio el comportamiento de las comunidades locales está dado por gente especializada en la agricultura, que han edificado una aldea ceremonial, integrado la costa con el valle, como dos opuestos complementarios. La población posiblemente se distribuyó en cinco nodos a lo largo del valle, donde el elemento clave para asentarse fue el agua. De esta manera, el éxito de la agricultura se habría fundamento en la captación del agua de vertiente, siendo conducida a través de acequias a los terrenos de cultivos.

En cuanto a la integración social, cuya mayor información la proporcionan los cementerios y las plazas, posiblemente el eje estuvo dado en las relaciones de intercambio económico y en los rituales de iniciación, de paso y muerte de un individuo. En dichos rituales los grupos del valle se integraron, lo que permitió fortalecer los lazos ceremoniales y de parentesco, lo que los habría llevado a conformar una comunidad con identidad propia cuyos fundamentos se hallan en su propia historia milenaria.

\section{El periodo Medio en la secuencia arqueológica de Arica, propuesta de una fase temprana a partir de nuevas dataciones y contextos culturales}

El análisis de los fechados indican que entre el 200 al 700 DC hay una población que ha consolidado un modelo de vida aldeano basado en una agricultura de ambiente semi-tropical, la que se sustentó producto de la presencias de agua de vertientes y humedales que irrigaron determinadas áreas agrícolas en el valle (Muñoz y Zalaquett 2015). Esta situación, habría demandado una mayor planificación en cuanto a las áreas cultivables, haciendo más complejos los sistemas agrícolas en relación a la canalización, especialmente los ubicados en terrazas fluviales como Pampa de Alto Ramírez.

El escenario cultural y económico planteado en el párrafo anterior nos sugiere de un proceso con raíces similares, pero mucho más complejo y desarrollado de lo que conocemos como periodo Formativo clásico, en relación con la fase tardía denominada Alto Ramírez (Muñoz 2004). Por lo tanto, más que un proceso social vinculado a estructuras socioeconómicas formativas parece más bien relacionado al desarrollo de las comunidades agrícolas que caracterizarían el periodo Medio, en su fase inicial y a la cual denominamos "fase San Lorenzo" según Muñoz (2017), la que se caracterizaría por los siguientes indicadores: (1) planificación y organización de los asentamientos en el valle, (2) consolidación del trabajo de la tierra a través de líneas productivas de cultivo, (3) establecimientos permanentes en el Valle de Azapa, a través de aldeas, vinculadas a recursos hídricos, (4) desarrollo de tecnología hidráulica, a partir de la explotación de vertientes, (5) presencia de rebaños de la especie camélido (llamas y alpacas) en el valle, (6) distribución a lo largo del valle de distintos tipos de cementerios, en los cuales además, se observa tres tipos de tumbas en fosas (cuerpo protegido con una base cistada de piedra; cuerpo protegido por una estructura elevada, conformada por maderos y piedras; cuerpos depositados en canastos), (7) desarrollo de artesanías especializadas en textilería, trabajo en fibra vegetal y madera, confección de objetos en barro (no cocido) y en menor presencia el trabajo en piedra. Un aspecto destacable, por su alto nivel de especialización es el trabajo en miniatura, expresado en la confección de cestos, textiles y pequeñas piezas confeccionadas en barro con formas de ollas.

De lo anteriormente planteado el periodo Medio tendría dos fases, la primera como lo señalara anteriormente definida como Fase San Lorenzo, cuyo rango de edad se situaría entre los 300 al 650 DC aproximadamente, y la segunda representada por la fase Maitas-Chiribaya, con un rango de edad que iría entre los 700 al 950 DC. Esta última tendría sus fundamentos a partir de la definición dada por Dauelsberg (1972/1973). Sin embargo, es necesario considerar la presencia mayoritaria de cerámica decorada representada por una variedad de estilos propios de los valles costeros como Cabuza y Maitas-Chiribaya, acompañados en menor cantidad por estilos como Charcollo y Taltape y los de origen altiplano como Tiwanaku, con sus formas de tazón, keros, pucos y jarras.

De acuerdo con las fechas, el estilo Cabuza sería tardío, a partir del 900 DC, como plantaron tempranamente Focacci (1982), Schiappacasse et al. (1991), Cassman 
(1997), y recientemente Korpisaari et al. (2014). Sin embargo, como señalan Espoueys et al. (1995), Focacci (1982), Muñoz (2004), Rivera (1976), Uribe y Agüero (2001), entre otros, los contactos entre la costa del Pacífico y el altiplano Circumtiticaca se dieron desde antes de la Era Cristiana con la presencia de piezas y bienes exóticos entre ellos, collares, textiles, metales, etc., llevando entre sus motivos algunos de ellos, incluso la representación de la figura del sacrificador. Es probable que todas estas piezas fueron parte del intercambio que pudo haberse realizado durante los primeros siete siglos de la Era Cristiana. Sin embargo, no habría constituido un dominio ideológico, por parte de grupos externos vinculados con la cultura Tiwanaku en el Valle de Azapa.

Los indicadores culturales que atribuimos a ambas fases culturales hablan de una sociedad bien establecida en el valle, con el auspicio de un sistema agrícola fundamentado en un complejo sistema de irrigación por vertientes, donde además se logra consolidar una arquitectura propia de estos valles como lo refleja la aldea de San Lorenzo, espacio que aglutinó política, cívica y religiosamente a la población del valle, simbolizando, además, el germen de una identidad local que madurara en la Cultura Arica.

\section{Comentarios Finales}

Los datos cronológicos reconocen que las poblaciones locales de tradición valluna a inicios del periodo Medio fueron determinantes en la planificación de la vida aldeana en el Valle de Azapa. Los restos de cultura material a través de manufacturas y diseños usados en la vida cotidiana, así como en las ceremonias fúnebres celebradas, sugieren que fue una población que se movió con sus propios ejes conductuales, como consecuencia de una larga tradición cultural. Pensamos que esta forma de organizarse e interactuar fue producto de un sistema basado en la reciprocidad, complementariedad y conocimiento de los espacios que ofrecía el valle y su entorno, lo cual derivó de un manejo íntegro del espacio productivo del valle cuya economía central estuvo dada por la agricultura.

Las poblaciones azapeñas del periodo Medio constituyeron la estructura basal sobre el cual emerge la Cultura Arica, a fines de este periodo, el que se va a caracterizar por una identidad propia. Al parecer todas las experiencias previas logradas en el trabajo agrícola, conjuntamente con el aporte de los recursos del mar y los camélidos, habrían contribuido económicamente a la emergencia de esta Cultura Regional permitiendo un mayor aumento de la población en el Valle de Azapa con una estructura social y arquitectura más compleja, como lo demostraría la aldea de San Lorenzo en su etapa final (Muñoz y Gordillo 2016).

Agradecimientos: Este artículo es producto de la investigación desarrollada en el proyecto Fondecyt 1130249 y UTA 3736-18. Se agradece la colaboración del Sr. Ing. Manuel Rodríguez por el análisis estadístico de los fechados realizados, Sr. José Rocha y Sra. Mariela Santos por el material fotográfico que ilustran el presente texto y los evaluadores del manuscrito quienes entregaron observaciones acertadas para mejorar las ideas planteadas en el texto.

\section{Referencias Citadas}

Berenguer, J. 1988. Acerca de los materiales Loreto Viejo de Arica y la colonización del valle de Azapa por Tiwanaku. Ponencia presentada en el XI Congreso de Arqueología Chilena, 11-15 de Octubre, Santiago.

Berenguer, J. 2000. Tiwanaku. Señores del Lago Sagrado. Museo Chileno de Arte Precolombino / Banco Santiago, Santiago.

Berenguer, J. y P. Dauelsberg 1989. El norte grande en la órbita de Tiwanaku (400 a 1200 d.C.). En Culturas de Chile. Prehistoria. Desde sus Orígenes hasta los Albores de la Conquista, editado por J. Hidalgo, V. Schiappacasse, H. Niemeyer, C. Aldunate e I. Solimano, pp.129-180. Editorial Andrés Bello, Santiago.

Cassman, V. 1997. A Reconsideration of Prehistoric Ethnicity and Stat in Northern Chile: The Textile Evidence. Ph.D. dissertation, Arizona State University. University Microfilms, Ann Arbor.

Chacama, J. 2004. El Horizonte Medio en los Valles Occidentales del norte de Chile. Chungara Revista de Antropología Chilena Vol. Especial 1:227-233.
Dauelsberg, P. 1961. Algunos problemas sobre la cerámica de Arica. Boletín de Museo Regional de Arica 5:7-17.

Dauelsberg, P. 1972-1973. Sobre la problemática arqueológica de Arica. Carta respuesta a Luis Guillermo Lumbreras. Chungara Revista de Antropología Chilena 1 y 2:32-37.

Dauelsberg, P. 1985. Desarrollo Regional en los valles costeros del norte de Chile. Diálogo Andino 4:277-285.

Espoueys, O., M. Uribe, A. Roman y A. Deza 1995. Nuevos fechados por termoluminiscencia para cerámica funeraria del Período Medio del valle de Azapa ( $1^{\circ}$ Parte). Actas XIII Congreso Nacional de Arqueología Chilena Vol. 2, pp. 31-54.

Focacci, G. 1982. Nuevos fechados para la época del Tiahuanaco en la arqueología del norte de Chile. Chungara Revista de Antropología Chilena 8:63-78.

Focacci, G. 1990. Excavaciones arqueológicas en el cementerio Az-6, valle de Azapa. Chungara Revista de Antropología Chilena 24-25:69-124. 
Goldstein, P. 1995/1996. Tiwanaku settlement patterns of the Azapa valley, Chile. New data, and the legacy of Percy Dauelsberg. Diálogo Andino 14/15:57-73.

Goldstein, P. 2006. Andean Diaspora: The Tiwanaku Colonies and the Origins of South American Empire. Florida University Press, Florida

Korpisaari, A. y J. Chacama 2015. Introducción. En El Horizonte Medio: Nuevos Aportes para el Sur del Perú, Norte de Chile y Bolivia editado por A. Korpisaari y J. Chacama, pp. 9-28. Instituto Francés de Estudios Andinos, Travaux de l'Institut Français d'Études Andines y Universidad de Tarapacá, Colección Universidad de Tarapacá, Arica.

Korpisaari, A., M. Oinonen y J. Chacama 2014. A reevaluation of the absolute chronology of Cabuza and related ceramic styles of the Azapa valley, northern Chile. Latin American Antiquity 25 (4):409-426

Muñoz I. 1983. El poblamiento aldeano en el valle de Azapa y su vinculación con Tiwanaku (Arica-Chile). Documentos de Trabajo 3:43-93. Universidad de Tarapacá, Arica.

Muñoz, I. 1995/1996. Poblamiento humano y relaciones interculturales en el valle de Azapa: Nuevos hallazgos en torno al periodo Formativo y Tiwanaku. Diálogo Andino 14-15:241-278.

Muñoz, I. 2004. Estrategias de Organización Prehispánicas en Azapa: El Impacto de la Agricultura en un Valle del Desierto Costero del Pacífico. Ediciones Universidad de Tarapacá, Arica.

Muñoz, I. 2014. Aproximaciones al individuo, su entorno y cultura material. Conclusiones y comentarios finales. En Mil años de Historia de los Constructores de Túmulos de los Valles Desérticos de Arica: Paisaje, Monumentos y Memoria, editado por I. Muñoz y M.S. Fernández, pp. 229-240. Ediciones Universidad de Tarapacá, Santiago.

Muñoz, I. 2017. Espacios fúnebres, prácticas mortuorias y cronología en el cementerio Azapa-115: Aproximaciones en torno a la estructura social de los agricultores prehispánicos del Periodo Medio. Estudios Atacameños. Arqueología y Antropología Surandinas 54:5-36.

Muñoz, I., C. Agüero y D. Valenzuela 2016. Poblaciones prehispánicas de los Valles Occidentales del norte de Chile: desde el Periodo Formativo al Intermedio Tardío (ca. 1.000 años a.C. a 1.400 años d.C.). En Prehistoria en Chile. Desde sus Primeros Habitantes hasta los Incas, editado por F. Falabella, M. Uribe, L. Sanhueza, C. Aldunate y J. Hidalgo, pp. 181-237. Editoria Universitaria y Sociedad Chilena de Arqueología, Santiago.

Muñoz, I. y J. Chacama 2006. Complejidad Social en las Alturas de Arica: Territorio, Etnicidad y Vinculación con el Estado Inca. Ediciones Universidad de Tarapacá, Arica.

Muñoz, I. y G. Focacci 1985. San Lorenzo: Testimonio de una comunidad de agricultores y pescadores post-Tiwanaku en el valle de Azapa (Arica-Chile). Chungara Revista de Antropología Chilena 15:7-30.

Muñoz, I. y J. Gordillo 2016. Organización del espacio y uso de los recursos naturales en la conformación de aldeas y campamentos en e Período Medio en los valles de Azapa, norte de Chile y Caplina, sur del Perú. Chungara Revista de Antropología Chilena 48 (4):531-555.

Muñoz, I. y M. Peña 2018. El asentamiento prehispánico de San Lorenzo: Arquitectura y Paisaje del periodo Medio en el valle de Azapa (norte de Chile). Chungara Revista de Antropología Chilena 50 (1):5-28.
Muñoz, I. y F. Zalaquett 2015. El paisaje en el ordenamiento territorial prehispánico durante el Período Medio, valle de Azapa, norte de Chile. Revista de Geografía Norte Grande 60:21-62.

Ogalde, J.P. 2015. Análisis químico y problematización técnica de los pigmentos en la tradición alfarera Cabuza del valle de Azapa, norte de Chile. En El Horizonte Medio: Nuevos Aportes para el Sur del Perú, Norte de Chile y Bolivia, editado por A. Korpisaari y J. Chacama, pp. 227-246. Instituto Francés de Estudios Andinos, Travaux de l'Institut Français d'Études Andines y Universidad de Tarapacá, Colección Universidad de Tarapacá, Arica.

Parssinën, M. 2015. Desde la expansión de Tiwanaku hasta la diáspora postiwanaku: reflexiones finales. En El Horizonte Medio: Nuevos Aportes para el Sur del Perú, Norte de Chile y Bolivia, editado por A. Korpisaari y J. Chacama, pp. 297-330. Instituto Francés de Estudios Andinos, Travaux de l'Institut Français d'Études Andines y Universidad de Tarapacá, Colección Universidad de Tarapacá, Arica.

Ponce, C. 1971. Tiwanaku: Espacio, Tiempo y Cultura. Editorial Los Amigos del Libro, La Paz.

Rivera, M. 1976. Nuevos aportes sobre el desarrollo cultural altiplánico en los valles bajos del extremo norte de Chile durante el período Intermedio Temprano. En Homenaje al R.P. Gustavo Le Paige, editado por J.M. Casassas, pp.71-81. Universidad del Norte. Antofagasta.

Rivera, M. 1980. Cronología absoluta y desarrollo cultural prehispánico en el norte árido de Chile: Un método numérico para construir períodos arqueológicos. Temas Antropológicos del Norte de Chile. Estudios Arqueológicos Número Especial, pp. 146-165.

Rivera, M. 1983. Patrones prehistóricos y contemporáneos del uso de la tierra en el valle de Azapa, norte de Chile. Dialogo Andino 2:9-26.

Rivera, M. 1987. Tres fechados radiométricos de Pampa Alto Ramírez, Norte de Chile. Chungara Revista de Antropología Chilena 18:7-13.

Rivera, M. 2002. Historias del Desierto. Arqueología del Norte de Chile. Editorial del Norte, La Serena.

Rowe, J. 1962. Stages and periods in archaeological interpretation. Southwestern Journal of Anthropology 18 (1).

Santoro, C., A. Romero, V. Standen y A. Torres 2004. Continuidad y cambio en las comunidades locales, períodos Intermedio Tardío y Tardío, Valles Occidentales del Área Centro Sur Andina. Chungara Revista de Antropología Chilena 36 (1):235-247.

Schiappacasse, V., A. Román, I. Muñoz, A. Deza y G. Focacci 1991. Cronología por Termoluminiscencia de la cerámica del extremo norte de Chile. Primera Parte. Actas del XI Congreso Nacional de Arqueología Chilena. Tomo II, pp. 43-60, Santiago.

Sutter, R. 2006. Modelos alternativos para explicar el poblamiento prehistórico del valle de Azapa, norte de Chile, mediante análisis de correlación de matrices. Chungara Revista de Antropología Chilena 38 (1):63-82.

Ulloa, L. 1982. Evolución de la Industria textil prehispánica en la zona de Arica. Chungara Revista de Antropología Chilena 8:97-107.

Uribe. M. 1999. La cerámica de Arica 40 años después de Dauelsberg. Chungara Revista de Antropología Chilena 31 (2):189-228.

Uribe, M. y C. Agüero 2001. Alfarería, textiles y la integración del Norte Grande de Chile a Tiwanaku. Boletín de Arqueología PUCP 5:397-426. 


\section{Notas}

${ }^{1}$ Dauelsberg (1972/73) y Focacci (1982) señalan que Maitas y Chiribaya correspondería a una misma unidad estilística para el Valle de Azapa, situación que se repetiría en el valle de Caplina. Sin embargo, Muñoz y Gordillo (2016) señalan que el estilo Chiribaya tendría una mayor vigencia en el tiempo en los valles del sur de Perú.

${ }^{2}$ La fuente bibliográfica para la clasificación de Tiwanaku V fue tomada de los trabajos de Dauelsberg (1972/73), Focacci (1982) y Espoueys et al. (1995) quienes compararon la secuencia de Azapa con la planteada por Ponce (1971) para el área de Tiwanaku.

${ }^{3}$ En un trabajo reciente Ogalde (2015) señala diferencias químicas entre las muestras de recipientes tiwanakotas del altiplano y tiwanakotas del valle de Azapa (estilo Cabuza), lo cual demostraría que la cerámica Cabuza imita el estilo Tiwanaku, y por lo tanto, no se habría confeccionado en el altiplano.
${ }^{4}$ Parssinën (2015) señala que estas nuevas fechas radiocarbónicas dan a entender que la cerámica Cabuza floreció mayoritariamente después del colapso estatal de Tiwanaku lo que obliga a repensar el desarrollo cultural de Kolisuyo, en general, y del Valle de Azapa en particular.

${ }^{5}$ De las tradiciones cerámicas de Moquegua, llámese ChenChen y Tumilaca, algunos tiestos presentan similitudes con las tradiciones cerámicas de Azapa, como Cabuza y Loreto Viejo, contextos que se remontarían alrededor del 900 DC.

${ }^{6}$ Respecto al uso de fechados radiocarbónicos no calibrados, que en el caso del periodo Medio la calibración suele hacer los fechados aprox. 50-100 años más tardíos, este análisis se realizará cuando se traten los procesos específicos de cada fase del periodo Medio en Azapa. 
